\title{
Prevalence of thyroid dysfunction and thyroid autoimmunity in infertile women
}

\section{Original Article}

\author{
Ahmed Alaa-El-Din Wali', Walaa Abdelfattah', Shimaa Mostafa Abd-El-Fatah ${ }^{1}$ \\ ${ }^{1}$ Department of Obstetrics and Gynecology, ${ }^{2}$ Department of Clinical and Chemical pathology, \\ Faculty of Medicine, Cairo University, Egypt
}

\begin{abstract}
Aim: This study aimed to determine the prevalence of thyroid autoimmunity (antithyroglobulin and antithyroid peroxidase) and thyroid dysfunction (hypo- or hyperthyroidism) among women with different causes of infertility.

Materials and Methods: A cross-sectional study was carried out among 255 patients with infertility who were recruited from the gynecology clinic, Kasr Al Ainy Hospitals, Cairo University, Egypt. Patients were divided into 4 groups. Group A including infertile women with either unexplained or anovulatory infertility with/without associated male factor, group B including infertile women with a tubal (mechanical) factor of infertility with/without associated male factor, group $\mathrm{C}$ including infertile women with both anovulation and tubal factor with/without associated male factor and a control group including women with exclusive male factor. Serum levels of TSH, antithyroid peroxidase (anti-TPO) and antithyroglobulin (anti-TG) were measured in all patients.

Results: The TSH level was abnormal in 22 out of 255 women $(9.8 \%) .44(17.3 \%)$ were positive for anti-TPO antibodies, $26(10.2 \%)$ were positive for anti-TG antibodies. Seventy patients (27.5\%) had TSH levels above $2.5 \mathrm{mIU} / \mathrm{L}$. No significant difference was found between different causes of infertility in TSH or antithyroid antibodies. There was a positive correlation between frequencies of both positive anti-TG and secondary infertility in group B.

Conclusion: There is no significant relationship between thyroid dysfunction and the type of infertility (functional or mechanical), or its duration. According to our study, the prevalence of antithyroid antibodies seems to be the same in infertile women and control. Antithyroglobulin might be associated with secondary infertility due to tubal factor.
\end{abstract}

Key Words: antithyroid antibodies, antithyroglobulin, antithyroid peroxidase, infertility, thyroid autoimmunity

Received: 21 July 2020, Accepted: 27 July 2020

Corresponding Author:Ahmed Alaa-El-Din Wali, Department of Obstetrics and Gynecology, Faculty of Medicine, Cairo University, Egypt, Tel.: 01001735088, E-mail: ahmed.wali@kasralainy.edu.eg

ISSN: 2090-7265, November 2020, Vol. 10, No. 4

\section{INTRODUCTION}

Infertility is classically described as a disease of the reproductive system defined by the failure to achieve a clinical pregnancy after 12 months or more of regular unprotected sexual intercourse ${ }^{[1,2]}$. With an almost constant global prevalence of $10 \%-15 \%$, infertility, this condition carries medical, social and psychological burdens ${ }^{[3]}$.

A normal function of the thyroid gland is necessary for fertility; the hypothalamic-pituitary-thyroid axis is intertwined with the axis of hypothalamic-pituitaryovarian, with roles including secretion of folliclestimulating hormone (FSH) and luteinizing hormone (LH), ovarian steroidogenesis, prolactin secretion, folliculogenesis, oocyte maturation, granulosa cell activity, endometrial receptivity, trophoblastic differentiation and implantation $^{[4-11]}$.
Thyroid dysfunction is the second most common endocrine disorder following diabetes mellitus, affecting women in the childbearing age, and thyroid autoimmunity (TAI) is the most frequent autoimmune disorder in such women. In women in their reproductive age, the prevalence of hypothyroidism is estimated to be $2 \%-4 \%$, with a TAI level of $5 \%-20 \%{ }^{[12]}$. Moreover, $>20 \%$ of women having thyroid dysfunction suffer from an abnormal menstrual pattern, of which oligomenorrhea is the most famous irregularity ${ }^{[13]}$. Subclinical hypothyroidism has been linked to subfertility (mainly due to anovulation) in several studies $^{[1-16]}$.

Besides, overt hypothyroidism may cause spontaneous miscarriage, preeclampsia, preterm birth, placental abruption, and fetal demise ${ }^{[17,18]}$. TAI has been linked to unexplained infertility ${ }^{[19]}$, polycystic ovary syndrome ${ }^{[20]}$, premature ovarian failure ${ }^{[21]}$, endometriosis ${ }^{[21-23]}$, recurrent 
pregnancy $\operatorname{loss}^{[24]}$ and even poor outcomes in in-vitro fertilization resulting from higher rates of failure and miscarriage ${ }^{[25-27]}$

\section{AIM OF THE STUDY}

The objective of this study was to determine the prevalence of thyroid auto-immunity (the primary outcome) and thyroid dysfunction among women with different causes of infertility.

\section{PATIENTS AND METHODS}

Women with infertility attending the outpatient Gynecology and Infertility Clinics at Kasr Al-Ainy Hospitals, Cairo University, Egypt, were invited to participate in a cross-sectional study. The study was conducted between March 2016 and September 2016 and was approved by the institutional ethics committee. The principles laid down in the 2008 Helsinki Declaration update were followed.

The inclusion criteria were ages between 18 and 38 years, experiencing infertility, whether primary or secondary, regardless of the cause of infertility: functional, anatomical, male factor, unexplained, or combined factors. The exclusion criteria were known autoimmune diseases other than thyroid disorders (e.g., systemic lupus erythematosus, rheumatoid arthritis), women who work at nigh, an acute illness that requires hospitalization and being on any of the medications that may affect thyroid hormones levels such as steroids, dopamine, iodine, amiodarone, lithium, domperidone, thyroid hormone and phenytoin.

The sample size was calculated to be 186 participants, based on a margin of error of $5 \%$, level of confidence of $95 \%$, an estimated population of 30,000 and an expected prevalence of $14 \%$ of thyroid autoimmunity among infertile women, as found in a previous study ${ }^{[23]}$. Expecting a maximum dropout rate of $15 \%$, the sample size was increased to 225 participants.

Written informed consent was provided by all participants after the objective of the study had been explained, and they were ensured of confidentiality, as well as the ability to withdraw from the study at any time.

Next, a detailed medical history was taken from every participant regarding age; duration and type of infertility (primary or secondary); detailed menstrual, obstetric, contraceptive and sexual history; a history of galactorrhea, hirsutism, pelvic inflammatory disease, thyroid disorders, and any medical disorders; a history of previous investigations regarding infertility, e.g., hormonal profile, hysterosalpingography, history of prior surgeries, especially, laparotomies, and history of earlier interventions regarding infertility, e.g., laparoscopy, hysteroscopy, or assisted reproductive techniques. Routine clinical examination was performed, which included general abdominal and pelvic examinations, along with bimanual and speculum examination. Every candidate underwent a conventional two-dimensional transvaginal ultrasound, to determine the uterine size and shape, scan both ovaries, and possibly visualize hydrosalpinges. Each patient had their hormonal profile carried out by providing blood samples for day 2 serum FSH, LH, estradiol, and prolactin. The hormonal profiles done at our hospital up to three months before recruitment were not repeated. The ovulatory status of the participants was determined by a previously documented history of induction of ovulation with a good response, previously recorded ultrasonography, or previous estimation of midluteal serum progesterone. Tubal patency was determined according to a previous hysterosalpingography or laparoscopy. The presence of male factor was evaluated by a seminal fluid analysis from the partners done within the last three months from recruitment into this study.

According to the aforementioned infertility assessment, participants fitted into one of these three categories: Group A (Functional): with either unexplained or anovulatory infertility with/without associated male factor infertility, Group B (Mechanical): with a tubal factor of infertility with/without associated male factor infertility, Group C (Combined): with both anovulation and tubal factor with/ without the associated male factor of infertility. Participants with exclusive male factor infertility served as the control group.

The level of serum thyroid-stimulating hormone (TSH), antithyroglobulin (TG), and antithyroid peroxidase (TPO) were measured for all participating women. Blood was collected by sterile venipuncture on plain tubes containing separating gel, and serum was separated immediately by allowing the blood to clot $(60 \mathrm{~min})$ and centrifugation at $1500 \mathrm{~g}$ for $10 \mathrm{~min}$ to separate the cells from the serum and kept at $-20{ }^{\circ} \mathrm{C}$ until use. Quantitative determination of TSH, antithyroid (anti-TPO) antibodies and antithyroglobulin (anti-TG) antibodies was performed by the electrochemiluminescence immunoassay (ECLIA) technique that was intended for use on COBAS $\mathbb{R}$ immunoassay analyzers (COBAS-e 601) by using "Roche Diagnostics COBAS Elecsys ${ }^{\circledR}$ TSH 11731459122”, "Roche Diagnostics COBAS Elecsys ${ }^{\circledR}$ Anti-TPO antibodies 06368590190" and "Roche Diagnostics COBAS Elecsys ${ }^{\circledR}$ Anti-TG antibodies 06368697190”, all from "Roche Diagnostics GmbH, Sandhofer Strasse, Mannheim, Germany." The measuring ranges were 0.005 $100 \mathrm{mIU} / \mathrm{L}$ for TSH, 5-600 IU/mL for anti-TPO, and $10-4000 \mathrm{IU} / \mathrm{mL}$ for anti-TG antibodies. TSH levels were considered abnormal if below 0.27 or above $4.2 \mathrm{mIU} / \mathrm{L}$. Anti-TPO were deemed to be positive when above 34 IU/ $\mathrm{mL}$, and anti-TG when above $115 \mathrm{IU} / \mathrm{mL}$. 


\section{STATISTICAL ANALYSIS:}

Collected data were analyzed using IBM SPSS Statistics for Windows, version 23 (IBM Corp., Armonk, N.Y., USA). Data were explored for normality using Kolmogorov-Smirnov and Shapiro-Wilk tests. For parametric data, one-way ANOVA test was used to compare between more than 2 groups. Tukey's test was used for pairwise comparison between the groups when ANOVA test was significant. For non-parametric data, Kruskal-Wallis test was used to compare between more than 2 groups. Dunn's test was used for pair-wise comparison between the groups when Kruskal-Wallis test was significant. For testing the correlation between variables Spearman correlation test was used.

\section{RESULTS}

A total of 250 women were invited to participate in the study, 21 were excluded for not meeting the inclusion criteria and four withdrew from the study.

The participating 225 infertile women were divided into four groups (three study groups and one control group). Group A (Functional) consisting of 134 infertile women with either unexplained or an ovulatory infertility with/without associated male factor of infertility. Group B (Mechanical) consisting of 31 infertile women with tubal factor of infertility with/without associated male factor of infertility. Group C (Combined) consisting of 29 infertile women with both anovulation and tubal factor with/without associated male factor of infertility. The last group of 61 women with exclusive male factor infertility was used as a control group.
Participants' demographics and clinical data, including age, BMI, type of infertility, whether primary or secondary infertility and duration of infertility among the different groups, are shown in Table 1 . The mean age and BMI of the four groups were almost matched with minimal variation.

Out of the total study population $(\mathrm{n}=225), 22$ women $(9.8 \%)$ had abnormal TSH levels, 16 women (7.1\%) had an abnormally high TSH (above $4.2 \mathrm{mIU} / \mathrm{L}$ ), while $6(2.7 \%)$ had an abnormally low TSH (below $0.27 \mathrm{mIU} / \mathrm{L}$ ). When considering $2.5 \mathrm{mIU} / \mathrm{L}$ as a more strict cutoff value for defining high $\mathrm{TSH}$, it was found that 70 patients $(27.5 \%)$ have an elevated TSH level. With regards to antithyroid antibodies, $44(17.3 \%)$ were positive for anti-TPO antibodies, and $26(10.2 \%)$ for anti-TG antibodies.

No significant difference was found when TSH and antibody levels were correlated to participants' age or duration of infertility. The four groups were compared relative to the prevalence of hypothyroidism (high TSH) or hyperthyroidism (low TSH) and thyroid autoimmunity. There was no difference between the four groups regarding the prevalence of abnormal TSH levels (Table 2). Also, there was no significant difference among the groups with regards to the incidence of antiTPO and anti-TG. However, when the groups were further subdivided into primary and secondary infertility, a correlation was found within group B (Mechanical), as the frequency of secondary infertility increased, the frequency of positive anti-TG and anti-TPO increased. This correlation was statistically significant for anti-TG, but not for anti-TPO.

Table 1: Demographic characteristics of the study population

\begin{tabular}{|c|c|c|c|c|c|c|c|}
\hline & \multirow{2}{*}{$\begin{array}{l}\text { Age } \\
\text { (year) }\end{array}$} & \multirow{2}{*}{ BMI } & \multicolumn{5}{|c|}{ Infertility } \\
\hline & & & \multicolumn{2}{|c|}{ Primary } & \multicolumn{2}{|c|}{ Secondary } & \multirow{2}{*}{$\begin{array}{c}\begin{array}{c}\text { Duration } \\
\text { (year) }\end{array} \\
\text { Mean } \pm \mathrm{SD}\end{array}$} \\
\hline & Mean \pm SD & Mean \pm SD & Frequency & Percent & Frequency & Percent & \\
\hline Group A & $28.77 \pm 5.37$ & $29.94 \pm 7.35$ & 89 & 66.4 & 45 & 33.6 & $3.76 \pm 3.09$ \\
\hline Group B & $29.71 \pm 4.41$ & $28.39 \pm 5.40$ & 23 & 74.2 & 8 & 25.8 & $4.11 \pm 2.47$ \\
\hline Group C & $30 \pm 4.78$ & $29.54 \pm 5.59$ & 12 & 41.4 & 17 & 58.6 & $3.74 \pm 2.49$ \\
\hline Control & $28.93 \pm 5.07$ & $28.31 \pm 5.21$ & 46 & 75.1 & 15 & 24.9 & $4.42 \pm 3.65$ \\
\hline
\end{tabular}


Table 2: Serum TSH level among different groups

\begin{tabular}{|c|c|c|c|c|}
\hline TSH (mIU/L) & Groups & Frequency & Percentage & Pvalue \\
\hline \multirow{5}{*}{$>4.2$} & A & 10 & 7.5 & \multirow{5}{*}{0.2033} \\
\hline & B & 3 & 9.7 & \\
\hline & & & & \\
\hline & $\mathrm{C}$ & 1 & 3.4 & \\
\hline & Control & 2 & 3.3 & \\
\hline \multirow{5}{*}{$>2.5$} & A & 37 & 27.6 & \multirow{5}{*}{0.2419} \\
\hline & B & 8 & 25.8 & \\
\hline & & & & \\
\hline & $\mathrm{C}$ & 7 & 24.1 & \\
\hline & Control & 18 & 29.5 & \\
\hline \multirow{5}{*}{$<0.27$} & A & 4 & 3.0 & \multirow{5}{*}{0.5233} \\
\hline & B & 2 & 6.5 & \\
\hline & & & & \\
\hline & $\mathrm{C}$ & 0 & 0.0 & \\
\hline & Control & 0 & 0.0 & \\
\hline
\end{tabular}

$P$ value was considered significant if less than 0.05

\section{DISCUSSION}

The prevalence of TAI and thyroid dysfunction among infertile women has been addressed in several studies over the past decades. It is well known that their incidence is relatively high in women of reproductive age; however, there is still a conflict between studies regarding the definition of subclinical hypothyroidism and association of TAI with infertility.

In this study, $9.8 \%$ of infertile women had thyroid dysfunction in the form of abnormal TSH levels, $8.6 \%$ had an abnormally high TSH level (hypothyroidism) and $2.7 \%$ had an abnormally low TSH (hyperthyroidism). There was no difference in TSH levels among different causes of infertility. Previous studies have shown a variable prevalence of thyroid dysfunction in infertile women ranging from $2.3 \%-43 \%$. Bohnet et al., earlier study as far back as 1981 showed that 20 of $185(11 \%)$ infertile women had hypothyroidism, of whom eleven had inadequate mid-luteal progesterone ${ }^{[14]}$. However, a later study by Gerhard et al. reported the prevalence of thyroid dysfunction among infertility women as high as $43 \%$ (80 out of 185$)^{[22]}$. Lincoln et al. studied 704 infertile women in whom serum TSH levels were measured. Sixteen $(2.3 \%)$ had elevated
TSH levels and eleven of the sixteen (69\%) were anovulatory ${ }^{[28]}$. In a retrospective study conducted in Finland, the prevalence of hypothyroidism was 4\% (12 out of 299). This study by Arojoki et al. grouped patients into ovulatory, unexplained, male, and tubal factors of infertility, and no difference was observed among the groups regarding TSH levels ${ }^{[15]}$. Prevalence of hypothyroidism in infertile women in the studies by Grassi et al., Poppe et al., Raber et al. and Abalovich et al. was 4.6\%, 0.9\%, 34\%, and 10.2\% respectively ${ }^{[16,21,23,29]}$.

Although still not recommended, a few studies have proposed lowering the threshold of TSH for diagnosing subclinical hypothyroidism from the conventional 4-5 mIU/L to 2-2.5 mIU/L ${ }^{[30-34]}$. Recently, women with high normal TSH levels $(>2.5 \mathrm{mIU} / \mathrm{L})$ were found to have unfavorable lipid profile, oxidative damage of membrane lipids and low levels of mannanbinding lectin, all of which are associated to infertility and adverse pregnancy outcomes ${ }^{[32-34]}$. When using the lower threshold of $2.5 \mathrm{mIU} / \mathrm{L}, 70(27.5 \%)$ patients in this study could be considered hypothyroid.

A further handful of studies have addressed the prevalence of thyroid autoimmunity in the normal population. In a large American survey which 
investigated 17,353 persons, women between the age of 20 and 29 years who were free from thyroid disease showed a prevalence of $10.4 \%$ and $8.5 \%$ for anti-TPO and anti-TG respectively, and women aged 30 to 39 had a prevalence of $12.6 \%$ and $13.6 \%{ }^{[35]}$. An earlier Korean study by Lee et al. on 390 women, the prevalence of anti-TG was $5 \%{ }^{[36]}$. In a more recent survey, Amouzegar et al. measured anti-TPO antibodies in 3390 normal women, and 541 (16\%) were found positive $\mathrm{e}^{[37]}$.

Regarding TAI in this study population of 255 infertile women, 44 women $(17.3 \%)$ were positive for anti-TPO antibodies and $26(10.2 \%)$ for anti-TG antibodies. The study demonstrated that the prevalence of TAI did not differ between the three study groups and the control group, except for a significant positive correlation between the presence of anti-TG antibodies and the occurrence of secondary infertility in group B (Tubal factor of infertility).

Previous studies differ in showing an association between the presence of thyroid autoantibodies and infertility, several studies showing a significant association while others do not. The earliest study by Wilson et al. in 1975 on autoantibodies association with infertility, eight out of 77 infertile women had antimicrosomal and antithyroglobulin antibodies compared to eleven out of 77 matched controls, indicating no difference. Other antibodies of interest in the study showed a significant difference ${ }^{[38]}$. Similarly, Roussev et al. studied many immunological changes in women with different forms of reproductive failure. Several immunological tests were significantly higher in these women compared to controls, especially antithyroid antibodies, which were elevated in women with a history of miscarriage. However, in the 63 infertile women in the study, five had the antithyroid antibodies, compared to none in the 15 controls, which was not statistically significant ${ }^{[39]}$. The study of Kutteh et al. demonstrated similar results. Antithyroid antibodies were higher in women with recurrent miscarriage $(22.5 \%)$, but not in infertile women candidates for assisted reproduction (19.2\%), when compared to controls $(14.5 \%)^{[40]}$. Another study interested in the effect of several autoantibodies on infertility is that by Reimand et al., one of the tested antibodies was thyroid microsomal antibody (antiTPO), and there was no statistical difference between study $(2 / 108)$ and controls $(15 / 392)^{[41]}$

Contrary to the above-mentioned studies, other works suggest an association between TAI and infertility. Geva et al. compared the presence of the thyroid antibodies in three groups of women; 40 women with unexplained infertility, 40 with the mechanical factor of infertility, and 40 healthy controls. A high prevalence of thyroid antibodies in women with unexplained and mechanical infertility, compared with the healthy controls, but there was no difference between the two infertile groups ${ }^{[19]}$. Abnormal immunological tests in relation to reproductive failure were also investigated by Kaider et al. and several tests were positive in the patients compared with the control group. Moreover, when specifically comparing cases with antithyroid antibodies, there was a significant difference in the positive cases among infertile women (51/167), compared to those positive among fertile controls $(16 / 109)^{[42]}$. Poppe et al. investigated 438 infertile couples and 100 age-matched fertile controls for anti-TPO. In couples with female factor infertility, there was an increased risk of positive anti-TPO in the female partner, compared with the control population. When female infertility was further sub-grouped, the risk was even higher in infertility due to endometriosis, who showed an anti-TPO prevalence of $29 \%{ }^{[23]}$. Other studies that demonstrated a significant association of TAI to infertility are the studies by Gerhard et al. and Abalovich et al. In the former study prevalence of TAI in cases of endometriosis was $44 \%$ compared to $9 \%$ in controls ${ }^{[22]}$. The latter showed a prevalence of $25 \%$ against $14 \%{ }^{[21]}$. In this study, an association between anti-TG and secondary mechanical (tubal) infertility was found. This finding could be attributable to the possible presence of endometriosis in these patients.

It is believed that several factors have played a role in the discrepancy in conclusions reached by the aforementioned studies. These studies vary widely in their study design, settings, and sample sizes. Most studies are cross-sectional surveys or retrospective analyzing data from records. Some studies have controls while others do not, and studies vary in whether the controls are healthy women or not. Other factors include selection biases, different methods of assay of antibodies and hence reference ranges, the inclusion of other antibodies, inclusion of women with overt thyroid disturbances, and recruitment of women with different causes of infertility.

It was supposed that an ideal study designed to determine the incidence TAI in women with infertility should be a prospective one, including cohorts of women with different factors of infertility, compared to matched healthy controls, and women with overt thyroid disease should be excluded from the start.

Strengths and weaknesses : The strength of the survey lies in it being the first study to address TAI in infertile Egyptian women. However, it does not preclude weakness. First, it is a cross-sectional study as almost all studies linking thyroid disorders and TAI 
to infertility. Second, unfortunately, our study did not have healthy fertile volunteers to be a control group; instead, women with exclusive male factor infertility served as the control group.

\section{CONCLUSION}

Determination of the TSH level is essential while doing an infertility workup due to the association between thyroid dysfunction (especially hypothyroidism) and infertility. However, there is no significant relationship between thyroid dysfunction and the type of infertility (functional or mechanical), or its duration. According to our study, the prevalence of antithyroid antibodies seems to be the same in infertile women and the controls, except for ATG antibodies being higher in secondary infertility due to tubal factor.

\section{CONFLICT OF INTEREST}

There are no conflicts of interests.

\section{REFERENCES}

1. Zegers-Hochschild, F., Adamson, G. D., de Mouzon, J., Ishihara, O., Mansour, R., Nygren, K., et al. "International Committee for Monitoring Assisted Reproductive Technology (ICMART) and the World Health Organization (WHO) Revised Glossary of ART Terminology, 2009." Fertil. Steril. 2009, 92, 1520.

2. Zegers-Hochschild, F., Adamson, G. D., de Mouzon, J., Ishihara, O., Mansour, R., Nygren, $\mathrm{K}$, et al. "The International Committee for Monitoring Assisted Reproductive Technology (ICMART) and the World Health Organization (WHO) Revised Glossary on ART Terminology, 2009.” Hum. Reprod. 2009, 24, 2683

3. Mosher, W. D., Pratt, W. F. "Fecundity and Infertility in the United States: Incidence and Trends.” Fertil. Steril. 1991, 56, 192.

4. Thomas, R., Reid, R. L. "Thyroid Disease and Reproductive Dysfunction: A Review." Obstet. Gynecol. 1987, 70, 789.

5. Maruo, T., Matsuo, H., Mochizuki, M. "Thyroid Hormone as a Biological Amplifier of Differentiated Trophoblast Function in Early Pregnancy." Acta Endocrinol. 1991, 125, 58.

6. Cecconi, S., Rucci, N., Scaldaferri, M. L., Masciulli, M. P., Rossi, G., Moretti, C., et al. "Thyroid Hormone Effects on Mouse Oocyte
Maturation and Granulosa Cell Aromatase Activity 1." Endocrinology. 1999, 140, 1783.

7. Jones, R. L., Hannan, N. J., Kaitu'u, T. J., Zhang, J., Salamonsen, L. A. "Identification of Chemokines Important for Leukocyte Recruitment to the Human Endometrium at the Times of Embryo Implantation and Menstruation." J. Clin. Endocrinol. Metab. 2004, 89, 6155.

8. Wakim, A. N., Polizotto, S. L., Buffo, M. J., Marrero, M. A., Burholt, D. R. "Thyroid Hormones in Human Follicular Fluid and Thyroid Hormone Receptors in Human Granulosa Cells." Fertil. Steril. 1993, 59, 1187.

9. Aghajanova, L., Stavreus-Evers, A., Lindeberg, M., Landgren, B.-M., Sparre, L. S., Hovatta, O. "Thyroid-Stimulating Hormone Receptor and Thyroid Hormone Receptors Are Involved in Human Endometrial Physiology." Fertil. Steril. 2011, 95, 230 .

10. Zhang, C., Guo, L., Zhu, B., Feng, Y., Yu, S., An, N., Wang, X. 'Effects of 3, 5, 3'-Triiodothyronine (T3) and Follicle Stimulating Hormone on Apoptosis and Proliferation of Rat Ovarian Granulosa Cells.” Chin. J. Physiol. 2013, 56, 298.

11. Oki, N., Matsuo, H., Nakago, S., Murakoshi, H., Laoag-Fernandez, J. B., Maruo, T. "Effects of 3,5,3'-Triiodothyronine on the Invasive Potential and the Expression of Integrins and Matrix Metalloproteinases in Cultured Early Placental Extravillous Trophoblasts." J. Clin. Endocrinol. Metab. 2004, 89, 5213.

12. Poppe, K., Glinoer, D. "Thyroid Autoimmunity and Hypothyroidism before and during Pregnancy." Hum. Reprod. Update 2003, 9, 149.

13. Krassas, G. E. "Thyroid Disease and Female Reproduction.” Fertil. Steril. 2000, 74, 1063.

14. Bohnet, H. G., Fiedler, K., Leidenberger, F. A. "Subclinical Hypothyroidism and Infertility." Lancet. 1981, 2, 1278.

15. Arojoki, M., Jokimaa, V., Juuti, A., Koskinen, P., Irjala, K., Anttila, L. "Hypothyroidism among Infertile Women in Finland." Gynecol. Endocrinol. $2000,14,127$

16. Raber, W., Nowotny, P., Vytiska-Binstorfer, E., Vierhapper, H. "Thyroxine Treatment Modified in Infertile Women According to Thyroxine- 
Releasing Hormone Testing: 5 Year Followup of 283 Women Referred after Exclusion of Absolute Causes of Infertility." Hum. Reprod. $2003,18,707$.

17. Casey, B. M., Leveno, K. J. "Thyroid Disease in Pregnancy.” Obstet. Gynecol. 2006, 108, 1283.

18. Yazbeck, C. F., Sullivan, S. D. "Thyroid Disorders during Pregnancy." Med. Clin. North Am. 2012, 96, 235.

19. Geva, E., Lessing, J. B., Lerner-Geva, L., Azem, F., Yovel, I., Amit, A. "The Presence of Antithyroid Antibodies in Euthyroid Patients with Unexplained Infertility and Tubal Obstruction." Am. J. Reprod. Immunol. 1997, 37, 184.

20. Janssen, O. E., Mehlmauer, N., Hahn, S., Offner, A. H., Gärtner, R. "High Prevalence of Autoimmune Thyroiditis in Patients with Polycystic Ovary Syndrome.” Eur. J. Endocrinol. 2004, 150, 363.

21. Abalovich, M., Mitelberg, L., Allami, C., Gutierrez, S., Alcaraz, G., Otero, P., Levalle, O. "Subclinical Hypothyroidism and Thyroid Autoimmunity in Women with Infertility." Gynecol. Endocrinol. 2007, 23, 279 .

22. Gerhard, I., Becker, T., Eggert-Kruse, W., Klinga, K., Runnebaum, B. "Thyroid and Ovarian Function in Infertile Women.” Hum. Reprod. 1991, 6, 338.

23. Poppe, K., Glinoer, D., Van Steirteghem, A., Tournaye, H., Devroey, P., Schiettecatte, J., Velkeniers, B. "Thyroid Dysfunction and Autoimmunity in Infertile Women." Thyroid 2002, 12, 997 .

24. Thangaratinam, S., Tan, A., Knox, E., Kilby, M. D., Franklyn, J., Coomarasamy, A. "Association between Thyroid Autoantibodies and Miscarriage and Preterm Birth: Meta-Analysis of Evidence." BMJ. 2011, 342, d2616.

25. Bussen, S., Steck, T., Dietl, J. "Increased Prevalence of Thyroid Antibodies in Euthyroid Women with a History of Recurrent InVitro Fertilization Failure." Hum. Reprod. 2000, 15, 545 .

26. Poppe, K., Glinoer, D., Tournaye, H., Devroey, P., van Steirteghem, A., Kaufman, L., Velkeniers, B. "Assisted Reproduction and Thyroid
Autoimmunity: An Unfortunate Combination?" J. Clin. Endocrinol. Metab. 2003, 88, 4149.

27. Kim, C.-H., Ahn, J.-W., Kang, S. P., Kim, S.-H., Chae, H.-D., Kang, B.-M. "Effect of Levothyroxine Treatment on in Vitro Fertilization and Pregnancy Outcome in Infertile Women with Subclinical Hypothyroidism Undergoing in Vitro Fertilization/Intracytoplasmic Sperm Injection." Fertil. Steril. 2011, 95, 1650.

28. Lincoln, S. R., Ke, R. W., Kutteh, W. H. "Screening for Hypothyroidism in Infertile Women." J. Reprod. Med. 1999, 44, 455.

29. Grassi, G., Balsamo, A., Ansaldi, C., Balbo, A., Massobrio, M., Benedetto, C. "Thyroid Autoimmunity and Infertility." Gynecol. Endocrinol. 2001, 15, 389.

30. Wartofsky, L., Dickey, R. A. "The Evidence for a Narrower Thyrotropin Reference Range Is Compelling." J. Clin. Endocrinol. Metab. 2005, 90, 5483.

31. Brabant, G., Beck-Peccoz, P., Jarzab, B., Laurberg, P., Orgiazzi, J., Szabolcs, I., et al. "Is There a Need to Redefine the Upper Normal Limit of TSH?" Eur. J. Endocrinol. 2006, 154, 633.

32. Karbownik-Lewinska, M., Marcinkowska, M., Stepniak, J., Lewinski, A. "TSH $\geq 2.5 \mathrm{mIU} / 1$ is Associated with the Increased Oxidative Damage to Membrane Lipids in Women of Childbearing Age with Normal Thyroid Tests." Horm. Metab. Res. 2017, 49, 321-326.

33. Karbownik-Lewinska, M., Stepniak, J., Zurawska, A., Lewinski, A. "Less Favorable Lipid Profile and Higher Prevalence of Thyroid Antibodies in Women of Reproductive Age with High-Normal TSH-Retrospective Study." Int. J. Environ. Res. Public Health. 2020, 17, 1-11.

34. Karbownik-Lewinska, M., Stepniak, J., Marcinkowska, M., Krygier, A., Lewinski, A. "Correction to: High normal TSH is associated with lower mannan-binding lectin in women of childbearing age." BMC Endocr. Disord. 2020, 20, 82 .

35. Hollowell, J. G., Staehling, N. W., Flanders, W. D., Hannon, W. H., Gunter, E. W., Spencer, C. A., 
Braverman, L. E. "Serum TSH, T(4), and Thyroid Antibodies in the United States Population (1988 to 1994): National Health and Nutrition Examination Survey (NHANES III)." J. Clin. Endocrinol. Metab. 2002, 87, 489.

36. Lee, M. S., Lee, D. S., Han, J. S., Cho, B. Y., Koh, C. S., Lee, M. "The Prevalence of Antithyroid Autoantibodies in Normal Korean PopulationAge, Sex Distribution and Its Relation to Thyroid Function.” Korean J. Intern. Med. 1986, 1, 31.

37. Amouzegar, A., Gharibzadeh, S., Kazemian, E., Mehran, L., Tohidi, M., Azizi, F. "The Prevalence, Incidence and Natural Course of Positive Antithyroperoxidase Antibodies in a PopulationBased Study: Tehran Thyroid Study." PLoS One 2017, 12, e0169283.

38. Wilson, C., Elstein, M., Eade, O. ., Lloyd, R., Wright, R. "Smooth-Muscle Antibodies In Infertility.” Lancet. 1975, 306, 1238.
39. Roussev, R. G., Kaider, B. D., Price, D. E., Coulam, C. B. "Laboratory Evaluation of Women Experiencing Reproductive Failure." Am. J. Reprod. Immunol. 1996, 35, 415.

40. Kutteh, W. H., Yetman, D. L., Carr, A. C., Beck, L. A., Scott, R. T. "Increased Prevalence of Antithyroid Antibodies Identified in Women with Recurrent Pregnancy Loss but Not in Women Undergoing Assisted Reproduction." Fertil. Steril. 1999, 71, 843 .

41. Reimand, K., Talja, I., Metsküla, K., Kadastik, U., Matt, K., Uibo, R. "Autoantibody Studies of Female Patients with Reproductive Failure." J. Reprod. Immunol. 2001, 51, 167.

42. Kaider, A. S., Kaider, B. D., Janowicz, P. B., Roussev, R. G. "Immunodiagnostic Evaluation in Women with Reproductive Failure." Am. J. Reprod. Immunol. 1999, 42, 335. 